\title{
STOCHASTIC METHODS FOR THE PREDICTION OF COMPLEX MULTISCALE PHENOMENA
}

\author{
BY \\ JAMES GLIMM (The University at Stony Brook, Stony Brook, NY) \\ AND \\ DAVID SHARP (The Los Alamos National Laboratory, Los Alamos, NM)
}

\begin{abstract}
The purpose of this paper is to develop a general framework for the prediction of complex multiscale phenomena and to illustrate this framework through comparison to two examples of current interest to the authors. Prediction involves a two-step process of inverse prediction to describe the system, given observations of its behavior, and forward prediction, to specify system behavior, given its description.
\end{abstract}

1. Introduction. This paper is concerned with methods for the analysis and prediction of complex phenomena. The complexity of systems considered here results from the interaction of a number of subsystems, or within a single subsystem from the interaction of phenomena occurring on a number of length and/or time scales.

The analysis and prediction problem divides into two components, the forward problem and the inverse problem. The forward problem is to determine the solution describing system behavior, given the governing equations and initial data. The inverse problem is to determine the governing equations and initial data, given observations of the system. These two problems are linked in usage as the inverse problem, on the basis of current observations, and give the governing equations and data needed to solve the forward problem, which in turn makes predictions going beyond current observations.

Forward prediction is very different from inverse prediction by statistical inference. Forward prediction assumes complete data and gives a complete answer. Even in the stochastic case, the data and the answer for forward prediction are complete within a probabilistic framework. For statistical inference, to solve the inverse prediction problem,

Received November 19, 1997.

1991 Mathematics Subject Classification. Primary 62A15, 76E10, 76T05; Secondary 76M35, 76S05.

The first author was supported by the Applied Mathematics Subprogram of the U.S. Department of Energy DE-FG02-90ER25084, the Army Research Office, grant DAAL03-92-G-0185 and through the Mathematical Sciences Institute of Cornell University under subcontract to the University at Stony Brook, ARO contract number DAAH-049510414, and the National Science Foundation, grant DMS9500568.

The second author was supported by the U.S. Department of Energy. 
the data is observational and is not complete. As a result, the solution of the inverse problem will necessarily be probabilistic.

Maximum likelihood selection of the most probable model $m$ can give the appearance of a unique (hence deterministic) solution to the inverse problem. Assuming the forward problem is nonlinear, the maximum likelihood observations of the forward prediction, based on a posterior distribution of possible models $m$ is not the same as these same observations of the unique forward prediction based on the maximum likelihood model $m$. In fact, many different models and many different forward predictions may yield the same observations. Thus the multiplicity of models as well as their individual likelihood must be given weight in prediction. This is similar to the modeling of thermodynamics, in which at a finite temperature, the statistical equilibrium minimizes free energy rather than total energy, and so prefers states of high multiplicity as well as states of low energy. For this reason we regard the uniqueness of the model determination by maximum likelihood distribution as potentially spurious.

The probability-based solution to the inverse problem, which is the model specification or parameter determination step, is used as input to the forward prediction problem. Thus, answers to the two-stage inverse-forward prediction problem must be probabilistic. These answers are framed in terms of confidence intervals or conditional probabilities which specify the probability of some unknown (e.g., future) system property given current observations and system knowledge.

In this paper, we illustrate these ideas through their application to two specific problems: multiphase fluid mixing and flow in porous media. Among the many other fields of science to which the ideas presented here are relevant, we mention only weather prediction. Conceptually, one can regard climate statistics as defining a prior distribution, which can then be modified by (a) current observations and (b) current predictions based on simulations using initial data from an earlier time period. Laws of physics can be used to refine the data analysis. The governing equations contain rapid transients (gravity waves) which are largely absent in atmospheric flows. The solutions converge to a "slow manifold" on which these waves are absent. Thus, it may be desirable to constrain the initialization analysis to give data in the slow manifold. While this is not too difficult if the manifold is approximated by its tangent plane, so that linear methods apply, a fully nonlinear implementation of these ideas is more difficult [48]. For a current summary of work on the data assimilation problem for weather forecasting, see [12].

The complex phenomena that require an approach of the type developed here are typically nonlinear, stochastic, and multiscale in the sense that they comprise phenomena spanning a range of distinct length and/or time scales. Many of the major outstanding problems of science are of this type, including turbulence, properties of real materials, materials by design, multiphase flow, scale up for flow in porous media, opacity of dispersed material, and structural biology.

Complexity results not only from the interaction of time and length scales, but also from the interaction of many subsystems. We regard the full system as being characterized by a number of critical design points, for which the performance of subsystems can be specified, and which thus isolate the subsystem interaction through input and output specifications from the internal subsystem dynamics. This model applies not only to 
technology, such as the ignition system for an automobile with subsystems consisting of the ignition key, battery, spark plug, fuel injection, starter motor, etc., it also applies to science, with the central nervous system containing multitudes of neurons, axons and dendrites, calcium and potassium channels, synapses, neuro-transmitters, and sensory organs. For complexity that results from a large number of well-characterized interacting subsystems, schematic methods of analysis, such as probabilistic risk assessment, discrete event simulation, and combinatoric and stochastic optimization apply [40]. These methods are outside the scope of this paper. Thus we are concerned here with complex phenomena for which the coupling to the laws of physics within the subsystem dynamics is integral to the full system analysis.

The forward prediction problems of interest here are governed partly by the partial differential equations of classical physics and partly by stochastic and probabilistic models [36]. Examples of such stochastic methods include stochastic partial differential equations (SPDEs), function space integrals, moment expansions, averaged equations, and closure. Examples of multiscale methods include the above and, in addition, subgrid models, homogenization, direct numerical simulation (DNS), and the renormalization group (RNG).

A typical multiscale forward prediction problem is the quantitative description of phenomena on a macroscale as a result of a scientific model describing the microscale. Both the microscale and the macroscale descriptions contain unknown or uncertain quantities, such as force laws, and thermodynamic and nonlinear response functions.

The microscale description is typically nonlinear and, when viewed from the macroscale, it is also typically stochastic and complex. It will, of necessity, involve a very large number of degrees of freedom and it will often display chaotic behavior (sensitive dependence on initial conditions and other problem data). Multiscale complex phenomena may be either deterministic or stochastic, depending on the length scale from which they are examined. In passing from one length scale to the next (the micro-macro transition) the descriptions are often deterministic on both length scales, with an intervening stochastic theory which joins them. An example is the description of a gas by the classical laws of interparticle interaction as the micro theory, statistical physics as the stochastic theory, and thermodynamics as the macro theory, again deterministic. However, not all problems simplify to this extent. If the length scales are not well separated, or if the coupling between them is strong, then the micro to macro transition may remove some but not all of the microscale stochasticity, so that the macro theory, with all micro variables integrated out, remains stochastic.

Multiscale forward prediction problems, which are largely solved, include the passage from statistical physics to thermodynamics, the passage from the Boltzmann equation to the Navier-Stokes and Euler equations of fluid dynamics, the classical and semiclassical limits of quantum mechanics, and the renormalization group theories of critical phenomena in the statistical physics of phase transitions.

A typical inverse prediction problem is the maximum likelihood specification of parameters to fix theories on the basis of partial observations. We regard this specification as an idealized limit (zero temperature thermodynamics) of a more general formulation of 
the inverse problem, in which uncertain model parameters are described by a probability distribution (as in finite temperature thermodynamics).

The Bayesian framework is convenient in quantifying the added value of information from various sources within a probabilistic framework. Bayesian analysis is based upon a prior distribution, which is a probability that represents our state of knowledge of the initial and boundary data of the solution of the physics model. It also represents a probability model for the parameters in the physics model itself, to the extent that these are incompletely characterized (the usual case). The prior distribution is the probability for this model before some piece of data is supplied through observation: the posterior distribution is the probability as corrected by observation. A more systematic formulation of these ideas follows.

By a model, we mean the specification of all equations of physics, including physics parameters used in them, and all initial and boundary conditions needed to determine the solution of the equations uniquely. Let $m$ denote a model. The prior distribution is a probability $p(m)$ to represent a "current" assessment of uncertain knowledge of $m$. With no change of notation, we also use $p$ to denote a probability distribution relative to a measure $d m$ on the space of models. Now suppose additional observations $\mathcal{O}$ are made. The observations have uncertainty associated with them. The comparison of the observations with the solutions derived from the model will have additional uncertainty associated with uncertainties in the solution process. On this basis we introduce a probability $p(\mathcal{O} \mid m)$, which is the probability of the observation, based upon knowledge of the model $m$. The prediction problem is to assess the posterior probability, which is defined as $p(m \mid \mathcal{O})$. According to Bayes' theorem,

$$
p(m \mid \mathcal{O})=\frac{p(\mathcal{O} \mid m) p(m)}{\int p(\mathcal{O} \mid m) p(m) d m} .
$$

The Bayesian framework is convenient for our multiscale hierarchy of theories, as the posterior distribution constructed from one length scale and set of observations will become the prior distribution for the next length scale, with its new observations.

Let $f=f(m)$ be some functional of the solution $s=s(m)$, and thus of the model $m$ that generates it. A typical problem is to evaluate the expectation

$$
E(f)=\int f(m) p(m \mid \mathcal{O}) d m=\lim _{n \rightarrow \infty}(1 / n) \sum_{i=1}^{n} f\left(m_{i}\right)
$$

where the models $m_{i}$ are sampled from the posterior distribution. The right side of (2) is the Monte Carlo evaluation of the integral in (2) defining $E(f)$.

Markov chain Monte Carlo (MCMC) methods provide a computational framework for sampling from the posterior distribution. They are thus important in the practical implementation of the Bayesian approach, in the evaluation of (2), and in the determination of the maximum likelihood $m$ relative to the posterior distribution (1).

2. SPDEs and forward prediction. Assume a system governed by the conservation law

$$
\frac{\partial U}{\partial t}+\nabla F(U)=0
$$


and suppose that the solutions are highly variable in space or time, so that they are effectively chaotic or random. Chaos means that the solution has sensitive dependence on initial conditions, which implies that to obtain predictive results, the initial data must be modeled as a random variable. In this case, the solutions are explicitly random, so that $U=U(x, t, \omega)$, where $\omega \in \Omega$ is a random variable. Alternatively the solution boundary data may be random, or $F=F(U, x, t)$ may have a rapidly varying explicit space or time dependence, and thus be modeled as approximately random, or $F=F(U, x, t, \omega)$ itself may be explicitly random. A general introduction to the modeling issues relating chaos, randomness, and SPDEs can be found in [36].

We introduce an averaging process. This can be defined as either a local average in time or space, denoted by an overbar, or an average over an ensemble of similarly prepared realizations of the same problem, denoted by angle brackets. The identity of these averages, if valid, is called the ergodic hypothesis, $\bar{U}=\langle U\rangle$. Averaging the conservation law gives the equation

$$
\frac{\partial \bar{U}}{\partial t}+\nabla \overline{F(U)}=0 .
$$

This equation is not very useful as it stands because it does not close, i.e., because $\overline{F(U)} \neq F(\bar{U})$. Thus $\bar{F}$ is a new quantity, not defined in terms of the averaged conserved variables $\bar{U}$. Closure is the introduction of a new quantity $F_{\text {ren }}$, which satisfies the relation $F_{\text {ren }}(\bar{U}) \approx \overline{F(U)}$, so that

$$
\frac{\partial \bar{U}}{\partial t}+\nabla F_{\text {ren }}(\bar{U})=0
$$

Here, the approximation is specific to some context (flow regime, type of material microstructure, etc.) which defines the measure space $\Omega$ and the probability measure $d \omega$ on it. In the case of multiphase flow, the distinct regimes of slug flow and droplet flow are characterized by a difference of the coherence length scale for change from one phase to the other, and the measure $d \omega$ will thus be concentrated on flows $U$ that are slowly changing their phase structure in space for slug flow and doing so rapidly for droplet flow. In this context, an approximation is ideally an identity a.e. relative to $d \omega$ in some thermodynamic limit, but typically physically motivated approximations are preferred as they allow simpler, but still acceptable, closure laws.

In general $F$ will be approximated by polynomials in $U$, and because these polynomials serve to define $F_{\text {ren }}$, it would seem that allowing polynomials $\overline{U^{n}}$ as new dependent variables would solve the closure problem. However, the systematic derivation of equations for $\overline{U^{n}}$ introduces new unclosed flux terms $F_{n}$ at each order. For example, $\overline{U^{2}}$ satisfies the equation

$$
\frac{\partial \overline{U^{2}}}{\partial t}+2 \overline{U \nabla F(U)}=0
$$

Thus, the hierarchy of moment equations does not close at any finite order. In practice, truncation of the series at first or second order $(n=1,2)$ is all that yields useful results, due to the complexity of the higher-order closures, the excessive approximations, and the increasing number of unknown phenomenological parameters they contain. 
We assume that the problem of interest is described through a hierarchy of theories, each valid on a specific length scale. Thus, each provides an alternate description of what is fundamentally the same phenomena. A complete solution of the problem on any given length scale uniquely determines the answer on all larger ones, independently of the details contained in the solutions to the still smaller length scale problems. This reductionist statement is causality, with the time variable replaced by the operation of integration of small length scales and passage to theories described on larger ones. This new "time" is just the renormalization group "time" parameter. Based on this lengthscale causality we have the analog probability statement, which is the Markov property of statistical inference, that the present description of the physical state space (as a probability distribution) contains as much information regarding the "future", i.e., large length scales, as the complete knowledge of the past, i.e., all smaller length scales. Such an assumption is equivalent to the fact that the state, at the current length scale level of description, is a complete description of the system. It is thus sufficient to set initial conditions for the dynamical evolution. Applied to our inferential hierarchy of length scales, in a probabilistic sense, we see that the states of the system, indexed by length scale, form a Markov chain.

The hierarchical structure extends to time sequences and to a more general structure of subsystems separated by critical design points. Thus we generalize the Markov chain to an acyclic graph with directed edges, with state spaces at each vertex, and a Markovian axiom separating a "past" from a "future" defined by the inferential rules specified by the directed edges.

In addition to randomness in the specification of the model $m$, we want to regard the solution process itself having a random component as a method of modeling errors within the solution. Solution errors arise from two sources: numerical errors associated with discrete approximations in the numerical integration of the PDE's, and physics modeling approximations in the formulation of the PDE's. These errors are not associated with lack of data, but rather with requirements for a practical, if approximate, solution. Let $s_{a}(m)$ denote this approximate solution, and let $s_{e}(m)$ denote an idealized, but unknown, exact solution of the model $m$. The difference

$$
e_{s}(m)=s_{e}(m)-s_{a}(m)
$$

is the error. We regard the approximate solution method as fixed, and only denote explicitly in $e_{s}(m)$ the dependence of the error on the model. Thus for a model $m_{2}$ of difficulty greater (for example having stronger nonlinearities or more extreme parameters) than a model $m_{1}$, we expect that $e_{s}\left(m_{1}\right)<e_{s}\left(m_{2}\right)$.

We are concerned with errors that arise from fundamental or practical limitations of modeling, solutions, or observational processes. We thus assume that any predictable components of the errors have been removed. The resulting error $e_{s}$ is a deterministic function of $m$, but on the basis of the assumed absence of any predictable component, it is unknowable (in practice at least), and so we model it as a random process. The total solution error $e_{s}(m)$ will contain both highly variable components, with sensitive dependence on data, and systematic components, with smooth but also unknown dependence on data. Both components will be present in the random process that models the error. 
Accordingly, we assume that the random process, to the degree that it depends on $m$, does so smoothly. With this assumption, all sensitive dependence of the error on data is subsumed within the randomness of the error process.

To justify the application of this error analysis methodology to the computation of $E(f)$ in (2), we assume in addition that the $f$ in $(2)$ is insensitive to $e_{s}$. In the language of probability theory, we assume that the error $e_{s}(m)$, as a random variable defined on the space of models, is independent of the target function $f$ whose integral (2) is to be evaluated. In the case of thermodynamics, the target integrand $f$ should be a thermodynamic function of the ensemble. It is then independent of the stochastic fluctuations that occur in the ensemble, necessarily present in a stochastic computation of the ensemble. The alternative to this assumption is the statement that the solution error and the solution usage are strongly coupled. If this should occur, the correct scientific judgement would be that the solution needs to be improved, or its usage needs to be restricted, until the above modeling assumption is satisfied. For example, in weather forecasting, which may be given with a probability of error (as in the likelihood of rain tomorrow), the time period for the forecast and the level of detail offered are restricted so that the deterministic component of the prediction offers substantive content. Thus, long-range forecasts of up to 30 days are restricted to such statements as the averaged temperature or rainfall will be above or below long-term averages, five-day forecasts give summary descriptions of a day's weather (average temperature, degree of cloudiness or precipitation), while highly precise several-hour forecasts give precise locations of arrival of a storm system to within a few minutes at a given location.

Restating these ideas, we suppose that $f=f\left(s_{e}(m)\right)$ is actually a function of the solution $s_{e}(m)$. To justify the use of $s_{a}$ in the evaluation of (2), we must assume that the integral of $f\left(s_{e}(m)\right)-f\left(s_{a}(m)\right)$ with respect to the posterior distribution is small. Either the error $e_{s}(m)$ is concentrated in the solution modes that are independent of $f$, and thus leaves the values of $f$ unchanged, or it influences digits that are not significant in the confidence intervals with which $E(f)$ is reported. In other words, we are assuming that errors in the approximate forward solution are decoupled from the manner in which this solution is used. This is the normal requirement for scientific validity of the solution method $s_{a}$, but with validity criteria now made quantitative, relative both to observations $\mathcal{O}$ (see below) and to reported confidence in conclusions $E(f)$.

Similarly, we regard observational errors $e_{\mathcal{O}, \exp }$ as a random process, independent of the solution error process $e_{s}$. Combining these two independent processes with the solution $s_{a}$ and with simulated observations $\mathcal{O}\left(s_{a}\right)$ computed from the solution $s_{a}$ defines the probability $p(\mathcal{O} \mid m)$, as we now explain. Let $\mathcal{O}(s)$ be the value of the observation computed from the solution $s$. Assuming that this computation is exact, the error $e_{\mathcal{O} \text {,sim }}$

$$
e_{\mathcal{O}, \operatorname{sim}}=\mathcal{O}\left(s_{a}(m)+e_{s}\right)-\mathcal{O}\left(s_{a}(m)\right)
$$

associated with $\mathcal{O}(s)$ is due entirely to the error in $s$. The experimental observation of a value $\mathcal{O}$ implies that

$$
\mathcal{O}+e_{\mathcal{O}, \exp }=\mathcal{O}\left(s_{a}(m)\right)+e_{\mathcal{O}, \operatorname{sim}}
$$


and so with the definition

$$
\begin{gathered}
e_{\mathcal{O}, \text { tot }}=e_{\mathcal{O}, \text { sim }}-e_{\mathcal{O}, \exp } \\
p(\mathcal{O} \mid m)=p\left(e_{\mathcal{O}, \text { tot }}=\mathcal{O}-\mathcal{O}\left(s_{a}(m)\right)\right) .
\end{gathered}
$$

It is elementary to check that $e_{\mathcal{O}}$,tot has a probability density that is the convolution of the probability density of $e_{\mathcal{O}}$,sim with that of $-e_{\mathcal{O}, \exp }$.

Substitution of (11) in Bayes' formula (1) defines the posterior probability $p(m \mid \mathcal{O})$. In the idealized case of no experimental or simulation errors, $p(m \mid \mathcal{O})$ is a density defined on the level set $\mathcal{O}-\mathcal{O}\left(s_{a}(m)\right)=0$. The weight of this density depends on a model for the neglected measurement and simulation errors. For example, if we consider measurement errors as dominant $\left(s_{a}=s_{e}\right)$, and if the measurement errors (e.g, the size of the error bars) are independent of $m$ and thus of $s_{a}(m)$ and of the measurement sensitivity $\nabla_{m} \mathcal{O}\left(s_{a}(m)\right)$ of $\mathcal{O}\left(s_{a}(m)\right)$ with respect to $m$, then

$$
p(m \mid \mathcal{O})=\left.\left[\nabla_{m} \mathcal{O}\left(s_{a}(m)\right)\right]^{-1} p(m)\right|_{\mathcal{O}\left(s_{a}(m)\right)=\mathcal{O}}
$$

However, if the measurement errors are assumed to be proportional to the sensitivity $\left|\nabla_{m} \mathcal{O}\left(s_{a}(m)\right)\right|$, then $p(m \mid \mathcal{O})$ is just the trace of $p(m)$ restricted to the level set. Such a situation could arise if the measurement process displayed excess sensitivity to system variation (e.g., noise) and was filtered to smooth the data before being reported. Assuming that the errors are small, results in the posterior distribution being concentrated in a band near the level set $\mathcal{O}=\mathcal{O}\left(s_{a}(m)\right)$. Details of modeling of the errors will influence the posterior distribution, defined within this band.

Methods for quantifying errors due to numerical integration of differential equations are well understood. Solutions are tested under mesh refinement, comparison to analytic solutions, and to results of laboratory experiments. They are also tested by comparison to specialized, but more accurate, computations for idealized problems. These methods depend on the ability to solve the same or an analog problem more accurately, so that the comparison will estimate the size of the error of the original problem. In these tests, the requirement of efficiency of solutions can be dropped, which is the basis for achieving additional accuracy. Other methods of error analysis, such as a posteriori estimates (cf. $[52,72])$, Richardson extrapolation, and interval arithmetic, are evaluated within the construction of the numerical solution $s_{a}$.

Methods for quantifying errors due to incomplete representation of physics are less well understood, but follow the same pattern. Simple estimates result from order of magnitude considerations. More precise estimates require the ability to solve equations describing more complete physics. Since the error determination is done only rarely, the associated efficiency requirements are lower. If necessary, the comparison can be made in the context of an analog problem, to further reduce the computational burden of the extra physics.

We summarize this section by listing the five basic requirements that the two-stage prediction process places on the forward problem.

1. The solution $s_{a}(m)$ must be accurate, relative to its use in the evaluation of (2), so that the errors are (as an approximation) statistically independent of $f$ relative to the 
posterior distribution, or so that they are independent of the precision to which $E(f)$ is reported.

2. The solution operator $s_{a}(\cdot)$ must be similarly accurate relative to its use in construction of simulated observations $\mathcal{O}\left(s_{a}(m)\right)$, and to the allowed observational errors.

3. The solution $s_{a}(m)$ must be sufficiently efficient that repeated evaluations in the Monte Carlo evaluation of (2) are practical.

4. The solution operator $s_{a}(\cdot)$ must support a probabilistic framework for the description of the model $m$.

5. Error analysis must be given to quantify $e_{s}(m)$ as a random process.

3. Inference, MCMC, and the inverse problem. In discussing the inverse problem, we are concerned with statistical inference from incomplete data. We consider two typical problems. The first is the evaluation of the expectation (2). The second is the determination of the best choice of model $m$, given the observations $\mathcal{O}$.

Let us assume that the prior distribution is known, in the sense that it can be generated effectively (numerically if necessary). An inefficient generation of samples from the posterior distribution is to accept samples generated from the prior distribution with probability $p(\mathcal{O} \mid m)$. The MCMC method [23] for sampling the posterior distribution is to generate a stochastic process whose stable distribution is the posterior distribution, and to sample from this stochastic process directly. This process is given by the Metropolis-Hastings algorithm [39,23]. Given the current choice of the model $m_{i}$, let a trial successor $m^{\prime}$ be chosen from some process with a transition probability $q\left(m^{\prime} \mid m_{i}\right)$. This trial state is then accepted (i.e., $m_{i+1}=m^{\prime}$ ) with probability

$$
\alpha\left(m_{i}, m^{\prime}\right)=\min \left(1, \frac{p\left(m^{\prime} \mid \mathcal{O}\right) q\left(m_{i} \mid m^{\prime}\right)}{p\left(m_{i} \mid \mathcal{O}\right) q\left(m^{\prime} \mid m_{i}\right)}\right) .
$$

Otherwise, $m_{i+1}=m_{i}$. Since $p(m \mid \mathcal{O})$ enters (13) only as a ratio, evaluation of the denominator in (1) is not required, and we only require $p(\mathcal{O} \mid m)$ up to a normalizing constant. This unnormalized function is then referred to as a likelihood function, rather than a probability density.

Efficient modeling of high-dimensional integrals will require their decomposition into a number of lower-dimensional integrals, corresponding to subsystems of the original complex systems. As discussed above, the decomposition may be based on a hierarchy of length or time scales, or a time sequence, or on a subsystem decomposition of a full system. Let $m^{\nu}$ denote a lower-dimensional component of $m$, so that

$$
m=\left\{m^{\nu}: \nu \in N\right\},
$$

where $N$ is the index space for the component label $\nu$. For random fields, which are random functions of a spatial variable $\vec{x}$, the field value at $\vec{x}$ would define a further decomposition into components, beyond those suggested above.

The Gibbs sampler $[22,21]$ is a special case of the Metropolis-Hastings algorithm. It assumes a component decomposition (14). Its update proceeds component by component, and cycles over all components to achieve a single cycle of update for $m$. For each component, the update is to choose the expected value of that component, given current estimates of all other components. The acceptance probability $\alpha$ for the Gibbs sampler 
turns out to be one. For this method to work in practice, we must assume that the set $N$ of component indices has some structure, which defines neighborhoods in $N$. We then further assume that conditional expectations depend only on other components in a neighborhood of the component being updated.

The component decomposition (14) is the general framework of multivariate statistical inference [57]. Without assuming a neighborhood structure within $N$, we still require that the degree of joint correlations among the $m^{\nu}$ is limited. In practice, it is unusual to go much beyond pair correlations. We assume that triple (or perhaps quartic) and higher (truncated) correlations are zero in the sense that the higher correlations can be constructed explicitly from the lower-order correlations, and contain no new correlation information not present in the lower-order correlations. This assumption is a type of closure for the sequence of higher-order correlations, related to the moment expansions of $\S 2$.

An idealization of the posterior distribution for $m$ is to determine a single "best" choice of $m$, effectively replacing the posterior distribution by a single point mass measure. The maximum likelihood model $m$ is the one that maximizes the posterior distribution $p(m \mid \mathcal{O})$. Thus, optimization algorithms come into play, including gradient descent [6], simulated annealing [55, 46, 47], and genetic algorithms [41].

We summarize this section by listing the three requirements placed on the solution of the inverse problem.

1. The observations $\mathcal{O}$ must sufficiently concentrate the posterior distribution $p(m \mid \mathcal{O})$ to place the confidence intervals for (2) within allowed bounds.

2. The experimental error in $\mathcal{O}$ must be similarly small, and its contribution to $p(\mathcal{O} \mid m)$ must be quantified.

3. The MCMC or other statistical sampling method for the evaluation of (2) must be sufficiently efficient that numerical errors in the evaluation of (2) remain within allowed bounds.

4. Shock waves and fluid mixing. We consider a turbulent fluid mixing layer, with the mixing driven by acceleration across a density discontinuity, or by shear flow. The idealized cases of continuous acceleration, impulsive acceleration and pure shear flow define the Rayleigh-Taylor (RT), Richtmyer-Meshkov (RM), and Kelvin-Helmholtz (KH) instabilities respectively. This problem is stochastic in the sense that the fluid instability, active on many length scales, induces a sensitive dependence on initial conditions, i.e., chaos. In order to recover a well-posed dependence on initial conditions in the case of deterministic chaos, the problem is modeled using stochastic dynamics. It is multiscale in the sense that many different length scales are active in the problem at the same time [36].

The main requirements placed upon forward prediction can be summarized as accuracy, efficiency, and compatibility with a probabilistic framework. We address these issues by

1. DNS based on the Front Tracking code FronTier;

2. Modeling, also known as scale up, averaged equations, or subgrid models, based upon SPDEs and a probability ensemble of initial conditions; 


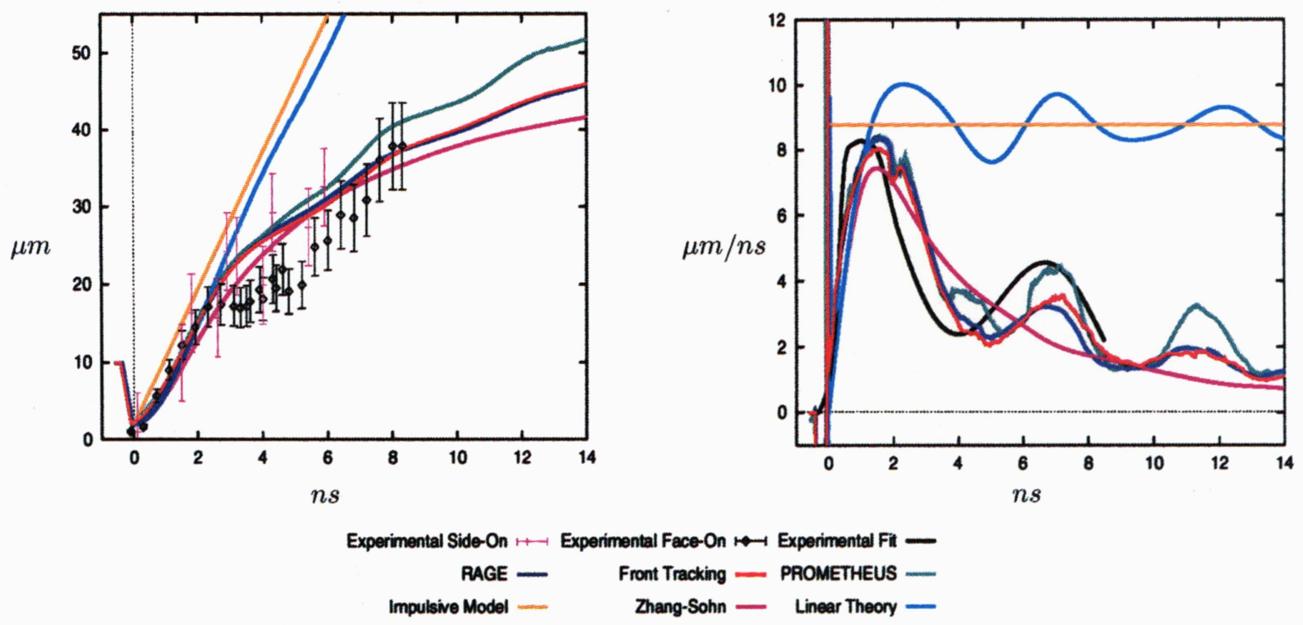

FIG. 1. Amplitudes and growth rates for Richtmyer-Meshkov instability induced by a Mach 15.3 shock refracting through a beryllium/foam interface. The initial perturbation had an amplitude of $4 \mu \mathrm{m}$ over a wavelength of $100 \mu \mathrm{m}$. The figure is taken from recent work by G. Dimonte (experimental data), B. Fryxell (PROMETHEUS simulation), J. W. Grove (FronTier), R. Holmes (FronTier and RAGE), R. P. Weaver (RAGE), Q. Zhang and Sohn (nonlinear model), and Y. Yang, Q. Zhang, and D. Sharp (linear theory).

3. Quantitative analysis of solution errors.

The main requirements for the inverse problem are good convergence properties for the Monte Carlo evaluation of integrals relative to the posterior distribution, and good precision in the choice of observations to limit the spread in the posterior distribution. These issues can be addressed by

4. Quantitative analysis of experimental errors;

5. Evaluation of the likelihood function $p(\mathcal{O} \mid m)$ on the basis of forward simulation of limited resolution observations of multiphase mixing data; and

6. MCMC algorithms, simulated annealing, and other methods of stochastic inference and optimization.

4.1. DNS for turbulent mixing. Highly-resolved direct numerical simulations (DNS) by the authors and colleagues have produced the first agreement between numerics and experiment for important acceleration-driven mixing problems [38, 42]. See Fig. 1. These computations used the hydro code FronTier. (For additional information, see

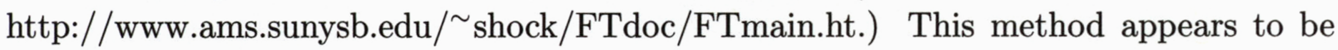
very satisfactory in the degree to which it is able to overcome the problem of mesh orientation dependence, and thus to allow valid computation of fluid instabilities in a curvilinear, for example cylindrical, geometry [67, 68]. See Fig. 2.

Front Tracking is a high-resolution method for the study of discontinuities and internal layers in the modeling of fluid flow and material deformation. It has recently been extended to three dimensions [25]; see Fig. 3 on p. 753. Recent improvements [24] in the 


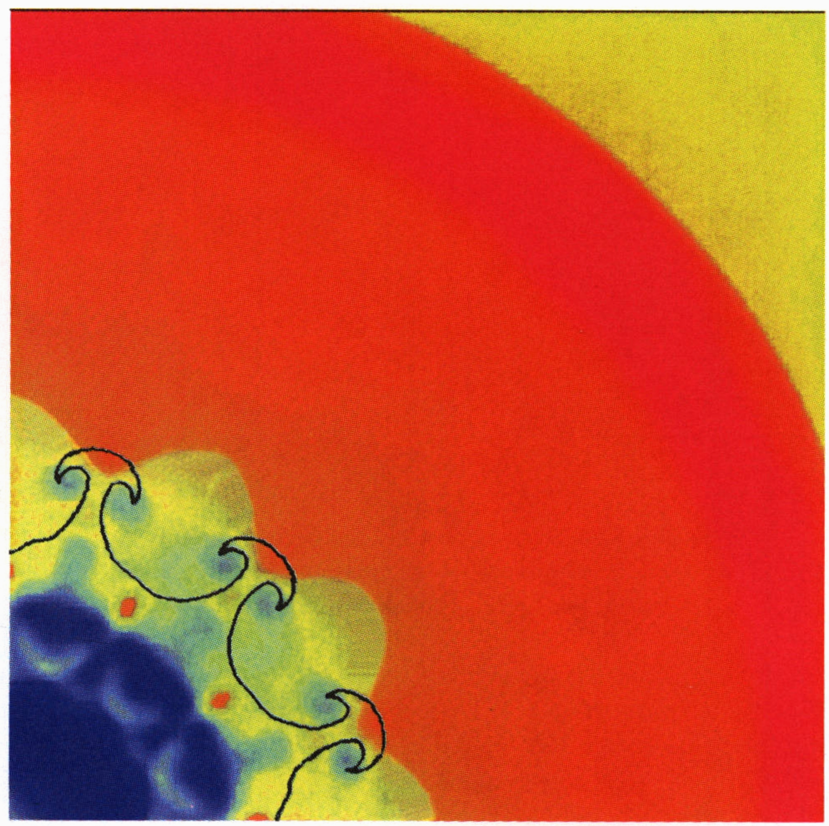

FIG. 2. Front Tracking simulation of a cylindrical shock-induced implosion. Shown are color contours of pressure. The dark line represents the position of the material interface. Computation by M. J. Graham and Q. Zhang

algorithm allow a grid-based interface description, which achieves a geometric simplicity and robustness comparable to the level set method, while retaining the high degree of fidelity in coupling to diverse physics which is a central strength of the Front Tracking method.

The importance of sharp resolution of solution discontinuities has led to a number of attacks on this problem. Of all the discontinuities, those associated with fluid or material boundaries are the most difficult computationally, because they lack the self-focusing nature of shock waves, and the numerical diffusion in the simulations tends to grow unacceptably with time. These methods go under the generic name of capturing, and those that address the interface problem specifically include volume of fluids, SLICK, artificial compression, and most recently the level set method. Each of these methods has a range of validity and class of problems for which they are applicable but in general, as the problems become more difficult (nonlinear, unstable, curvilinear, complex, etc.), the methods are observed to be unsatisfactory, and the search for new solutions to this problem continues. For example, the level set method [51] induces unphysical pressure waves at an accelerated fluid interface [25] if the two fluids are compressible and have different equations of state. Proposals to cure this deficiency within the level set framework appear to be difficult to distinguish from FT itself. In contrast, FT is the fundamentally exact representation of a discontinuity surface and has succeeded in correctly coupling to physics in a wide range of complex and difficult problems. 

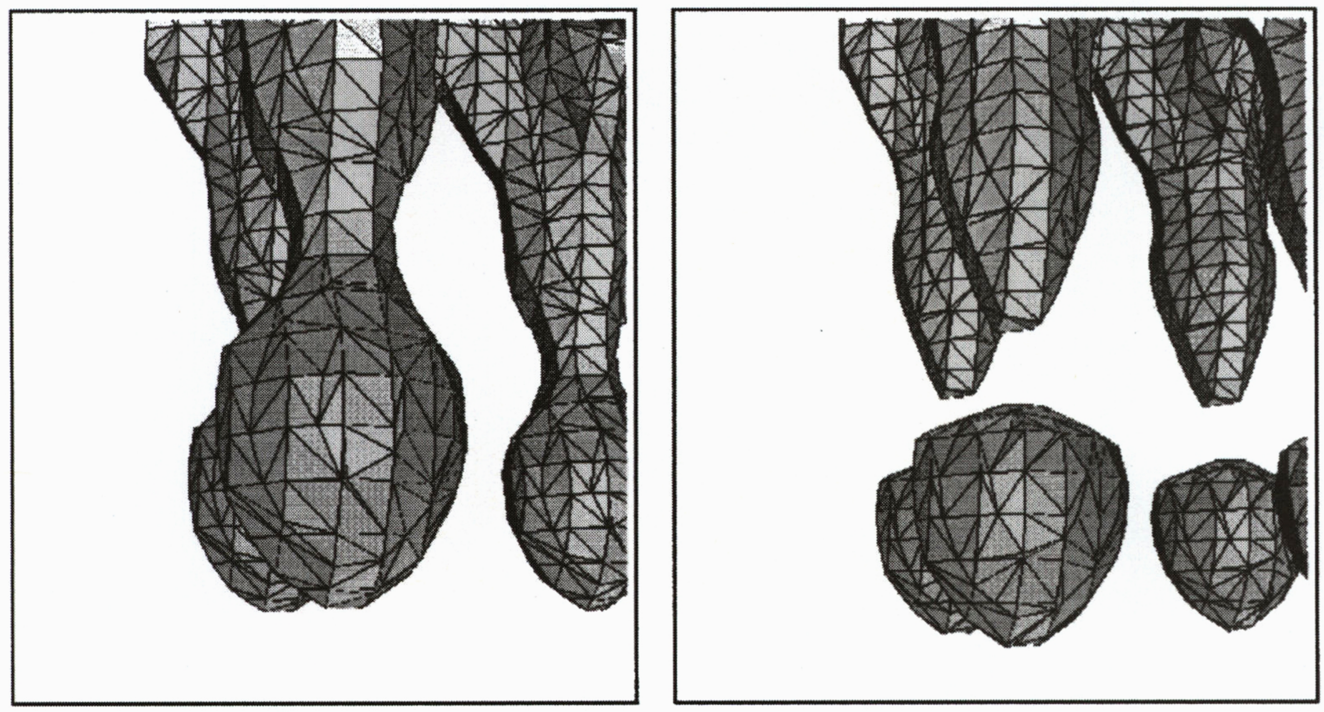

FIG. 3. A late stage in the RT instability in three dimensions, computed by the Front Tracking method. Shown here is pinch-off in a simulation with Atwood number $A=9 / 11$ at low compressibility. Simulation by Xiao Lin Li.

In our experience, the geometrical description of the interface is the easier problem, and the coupling of the interface to the physics is the more difficult problem. Thus we have chosen in FT the geometrical description that best supports coupling to interface physics.

FT has been questioned on two grounds. First, that it is not possible (in two dimensions, and more recently, as this was seen to be incorrect, in three dimensions). This claim has been shown to be false, by the constructive method of producing the implemented three-dimensional FT code FronTier, now in beta release to user communities. Secondly, FT has been described as being too complex. While its ability to get the right answer to problems of considerable difficulty rebuts this view, a further answer to the complexity issue lies in a recent FT development due to our collaborator X. L. Li. The grid-based interface description [24] and other recent algorithmic improvements, necessary to achieve satisfactory performance of FT in three dimensions, have considerably increased the simplicity and robustness of FT.

Theoretical developments kept pace with this progress and provide important, and very valuable, independent confirmation of the experimentally and numerically determined instability growth rates in both linear [63, 54] and nonlinear $[69,70,71]$ regimes.

4.2. Multiphase flow modeling. A new multiphase flow model [7, 29, 32] has been developed by the authors and colleagues. This model is totally hyperbolic (no complex eigenvalues), and thus is stable under numerical time integration. It supports distinct pressure and temperature fields when these are required by the physics. It couples to turbulence models in a natural fashion. It is derived systematically from the microphysical equations. This derivation is based on ensemble averaging, as discussed in $\S 2$. See also 


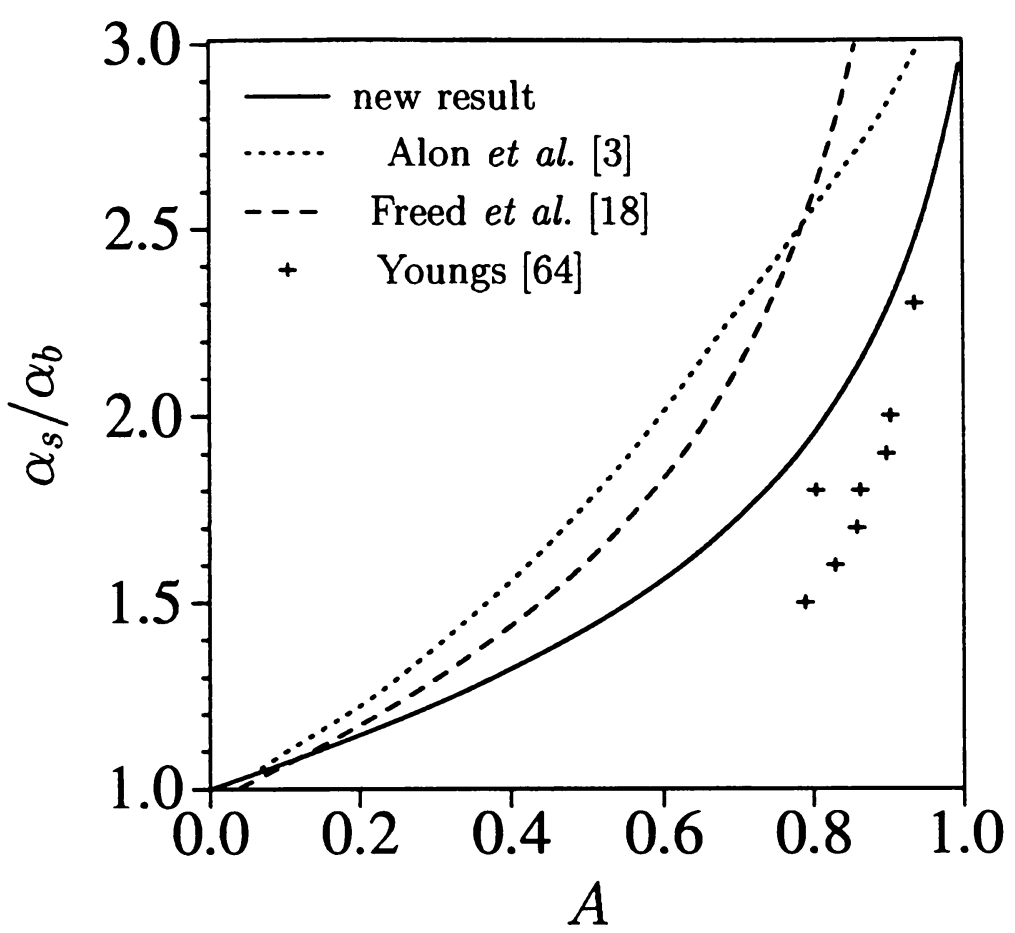

FIG. 4. Three analytic models are compared to experimental data. For RT mixing, the edge position $Z_{k}(t)$ of phase $k$ has an asymptotic form $Z_{k}(t)=\alpha_{k} A g t^{2}$, where $A$ is the Atwood number, $A=\left(\rho_{2}-\right.$ $\left.\rho_{1}\right) /\left(\rho_{2}+\rho_{1}\right)$, and $g$ is gravity. The figure shows the expansion ratio $\alpha_{2} / \alpha_{1}=Z_{2}(t) / Z_{1}(t)$ of the mixing zone as a function of the Atwood ratio $A$.

[36] and references cited there. The model does not require use of a (nonphysical) mixed fluid equation of state for mixtures that do not mix at the atomic level. The closure relations are simple and natural. For RT mixing layers, the model affords the best available prediction of the mixing zone edge expansion ratios. See Fig. 4. The model prediction for the volume fraction variation across an RT mixing zone agrees with experimental measurements $[64,59]$ at small Atwood number, as well as with RT numerical simulation data [7].

The equations that define this model resemble those of two independent fluids, with additional coupling terms. The fluids are distinguished by a subscript $k$, where $k=1$ and $k=2$ denote the light and heavy fluids, respectively. Let $\beta_{k}, \rho_{k}, v_{k}, p_{k}$, and $\varepsilon_{k}$ denote, respectively, the volume fraction, density, velocity, pressure, and specific internal energy of the fluid (phase) $k$. The equations are

$$
\frac{\partial \beta_{k}}{\partial t}+v^{*} \frac{\partial \beta_{k}}{\partial z}=0,
$$




$$
\begin{gathered}
\frac{\left(\partial \beta_{k} \rho_{k}\right)}{\partial t}+\frac{\partial\left(\beta_{k} \rho_{k} v_{k}\right)}{\partial z}=0 \\
\frac{\partial\left(\beta_{k} \rho_{k} v_{k}\right)}{\partial t}+\frac{\partial\left(\beta_{k} \rho_{k} v_{k} v_{k}\right)}{\partial z}=-\frac{\partial\left(\beta_{k} p_{k}\right)}{\partial z}+\beta_{k} \rho_{k} g+p^{*} \frac{\partial \beta_{k}}{\partial z} \\
\frac{\partial\left(\beta_{k} \rho_{k} \varepsilon_{k}\right)}{\partial t}+\frac{\partial\left(\beta_{k} \rho_{k} v_{k} \varepsilon_{k}\right)}{\partial z}=-p_{k} \frac{\partial\left(\beta_{k} v_{k}\right)}{\partial z}+(p v)^{*} \frac{\partial \beta_{k}}{\partial z}
\end{gathered}
$$

together with the constraint

$$
\beta_{1}+\beta_{2}=1
$$

A single fluid equation of state holds within each fluid. The quantities $v^{*}, p^{*}$, and $(p v)^{*}$ represent averages of microscopic quantities (products of primitive variables), which need to be modeled. Specifically, $a^{*}$ denotes the average of the fluid quantity $a$, conditioned on evaluation at the interface between the two materials; for example, $p^{*}$ is the average or expected interface pressure. Surface tension is neglected in this model, so that $p^{*}$ and $(p v)^{*}$ are uniquely defined quantities. In the presence of nonzero surface tension, the capillary pressure $p_{c}^{*}=p_{2}^{*}-p_{1}^{*}$ is an independent quantity to be modeled by closure.

A major new result gives the complete solution of this model, in the incompressible limit, in terms of boundary accelerations specified at the edge of the mixing zone [31, $33,30]$. The solution is given in closed form in terms of quadratures. Assuming constant acceleration-based scaling of the boundary velocities, as occurs for the RT problem, this solution exhibits renormalization group fixed-point behavior.

On the basis of general physical principles and a modeling assumption that $v^{*}$ (resp. $p^{*}$ ) depends only on $v_{1}$ and $v_{2}$ (resp. $p_{1}$ and $p_{2}$ ) and spatially dimensionless variables of the problem, the functional form of $v^{*}$ (resp. $p^{*}$ ) is constrained to be a convex linear combination of the single fluid velocities (resp. pressures) [30],

$$
\begin{aligned}
& v^{*}=\mu_{2}^{v} v_{1}+\mu_{1}^{v} v_{2}, \\
& p^{*}=\mu_{2}^{p} p_{1}+\mu_{1}^{p} p_{2},
\end{aligned}
$$

where the coefficients $\mu$ depend only on spatially dimensionless variables of the problem. We make the modeling assumption that each $\mu_{k}$ depends on $t$ and the volume fraction $\beta_{k}$ only.

The following explicit solutions for the model equations are valid:

$$
v_{k}=V_{k} \beta_{k^{\prime}} e^{-F_{k}\left(t, \beta_{k}\right)}
$$

where $V_{k}=\dot{Z}_{k}$ is the velocity of the edge $Z_{k}(t)$ of the mixing zone, defined as the locus of points at which the volume fraction of phase $k$ vanishes, and

$$
F_{k}\left(t, \beta_{k}\right)=\int_{0}^{\beta_{k}}\left[\frac{\mu_{k}^{v}\left(t, \phi_{k}\right)}{\phi_{k}}-\frac{\mu_{k^{\prime}}^{v}\left(t, \phi_{k^{\prime}}\right)}{\phi_{k^{\prime}}}\right] d \phi_{k} .
$$

In the above integration, the relation $\phi_{k}+\phi_{k^{\prime}}=1$ holds. Moreover, $\beta_{k}=\beta_{k}(z, t)$ is given as a solution of a scalar conservation law

$$
\frac{\partial \beta_{k}}{\partial t}+\frac{\partial}{\partial z} \mathcal{F}_{k}\left(t, \beta_{k}\right)=0
$$


where

$$
\mathcal{F}_{k}\left(t, \beta_{k}\right)=V_{k} \beta_{k} \beta_{k^{\prime}} e^{-F_{k}\left(t, \beta_{k}\right)} .
$$

Thus $\beta_{k}$ can be determined by integration along characteristics of (24). If the $\mu_{k}^{v}$ are independent of time, then the characteristic speeds are constant along characteristics, and given by $v^{*}$. Thus the characteristics are straight lines and $\beta_{k}$ is constant along characteristics in this case.

Assuming a fractional linear dependence of each $\mu_{k}$ on $\beta_{k}$, all parameters but one within the fractional linear expressions are completely fixed by physically required boundary conditions. The remaining parameter appears in $\mu_{k}^{p}$ and appears to be fixed by the Atwood number, which is a dimensionless measure of the density discontinuity. A closed form solution of the incompressible pressure equation (17) has also been obtained [30].

A simulation study of the compressible case is needed, comparing DNS simulation of the microphysics to numerical solutions of the averaged equations. Simulation is essential because we do not anticipate closed-form analytic solutions in the compressible case.

Boundary conditions at the edge of the mixing zone are needed to complete the closure of the multiphase flow equations. The missing boundary condition at the edge of each mixing zone is associated, in the compressible case, with incoming sound waves associated with the phase of the vanishing volume fraction entering that region. The number of variables and equations needed to describe the system changes at the mixing-zone boundary, and on the two-phase side of the boundary, is the single piece of characteristic information that is missing from the problem. This data is still missing in the incompressible limit, where the mixing-zone edge accelerations are shown rigorously to be free parameters in the closed-form solution. More generally, an exact identity (balance of forces) was derived among all boundary forces, in the incompressible limit, as a consequence of the closed-form pressure solution [30]. With all quantities evaluated at the mixing-zone edge $z=Z_{k}(t)$, we find

$$
(-1)^{k} \rho_{k} \ddot{Z}_{k}=\left(\rho_{2}-\rho_{1}\right) g-\frac{\partial\left(p_{2}-p_{1}\right)}{\partial z}-\rho_{k^{\prime}} V_{k}^{2} \frac{\partial \beta_{1}}{\partial z}+(-1)^{k}\left(\nu_{k}-1\right)\left(p_{2}-p_{1}\right) \frac{\partial \beta_{1}}{\partial z}
$$

where

$$
\nu_{k}(t)=\lim _{\beta_{k} \rightarrow 0} \frac{\mu_{k}^{p}\left(t, \beta_{k}\right)}{\beta_{k}} .
$$

This equation relates edge acceleration to form drag, with a rigorously defined drag coefficient, buoyancy, and two pressure difference terms.

Any additional relation (obtained, for example, by setting some terms to zero, and modifying the coefficients of others) will uniquely determine the edge acceleration, and thus close the system. Several phenomenological conditions have been proposed, [65, $32,3,4]$ as drag relations, to introduce new information into the boundary conditions at each mixing-zone edge, and thus to complete the closure of the system. Closure will depend on the flow regime, and as the number of regimes that are to be fit using a single closure increases, so does the required number of adjustable parameters. In this connection, we mention a zero-parameter determination of the RT mixing-zone bubble edge expansion rate. Agreement within $20 \%$ with experimental and simulation values 
was achieved for this simple theoretical model, based on a statistical ensemble of bubbles, whose dynamics is a combination of single bubble dynamics, pairwise bubble interactions constructed from bubble envelope modes, and bubble merger. The approximate solution of this model used renormalization group methods [34, 37, 66, 36]. This bubble merger model and methodology have since been adopted by other authors.

It is interesting to observe that self-similarity and scaling laws are inherent features of RNG fixed points and also of Riemann solutions for systems of conservation laws. In the present case, the RNG fixed point and the Riemann solutions describe the largetime asymptotic behavior of the solutions. A very general theory for conservation laws in one space dimension [8] states that the conservation law solution with general initial data converges to the self-similar solution with self-similar data given by the original data evaluated at $|x|=\infty$. This self-similar solution is called a Riemann solution. The reason for the identity of these two approaches to self-similarity is that the RNG time variable coincides with a reparameterized physical time. This occurs because the mixing process is dynamically self-similar with coarse graining, or evolution to larger scale structures, occurring through the process of bubble merger. We propose that it is the twodimensionality, i.e., the thinness, or high aspect ratio, of the mixing layer, that accounts for the dominant flow of information from small to large length scales. The occurrence of an RNG fixed point requires the absence of a dominant length scale. Compressibility introduces a length scale into the problem, namely that over which pressure differences induce density differences. It is for this reason that compressibility breaks the fixed-point behavior and thus we find RNG behavior in the incompressible limit only.

4.3. Observational data and the inverse problem for fluid mixing. MCMC methods [23] and other modern methods of statistical inference are appropriate tools to relate partial information to an overall probabilistic assessment of the state of the physical system. For fluids, the available types of observational data include:

1. A statistical characterization of initial surface perturbations;

2. Measurements of statistical fluid quantities, such as mixing-zone growth rates, volume fractions, mean densities, the distribution of particle sizes and shapes, and average velocities;

3. Frequency-dependent measured opacities of the multiphase mixture; and

4. Limited resolution tomographic data.

Observational data of this kind defines a sequence of stochastic state spaces. These are connected by SPDEs, through a mapping from initial data to a stochastic solution. Thus the Euler equation of fluid flow maps an initial surface distribution, specified stochastically, to a multiphase mixture described through a statistical ensemble. Use of refractive index matched fluids and laser-induced fluorescence allows a precise recording of interface positions along a thin slice taken out of a three-dimensional flow [56]. Due to the two-dimensional nature of the data, statistical methods of data analysis allow characterization of some but not all features that specify the three-dimensional interface geometry of the mixture statistically. Thus, from data collected from two-dimensional $x, z$ slices at constant values of $y$, scalar quantities such as volume fraction can be inferred, as can $x$, $z$ correlation tensors, while $x, y$ and $y, z$ correlation tensors cannot, unless the property of statistical homogeneity relative to rotations in the $(x, y)$-plane is satisfied. Numerical 
methods for statistical analysis of two-phase (binary) three-dimensional data have been developed $[60,49,17]$.

Data concerning correlation tensors for the multiphase distribution are required in the up-scaling of the radiation field in the diffusive approximation. Nonlinear interaction of the multiphase mixture with the radiation field can also be considered [50]. X-ray imagining provides a further measure of the multiphase volume fraction, but typically will not allow full three-dimensional shape reconstruction due to limitations in the number of independent tomographic axes.

5. Flow in porous media. Averaging over realizations sampled from the posterior distribution has been used in engineering practice for the prediction of engineering outcomes and the formulation of engineering decisions [10]. Bayesian statistical methods for history matching were used in a study of remediation of a groundwater contamination site $[1,2]$. The reference that comes closest to our point of view is [53]. Additional references are surveyed in $\S 5.2$ below.

5.1. Forward prediction and scale-up. For application of stochastic prediction methods to flow in porous media, we require an efficient and accurate solution method for the reservoir flow equations, consistent with a probability framework, and we require a quantitative analysis of solution errors. Accurate prediction of fluid flow requires specification of the geology at a very fine level of detail.

We can think of the probability modeling as a two-stage process, which begins a prior $p_{1}(m)$ distribution, assumed known from generic geostatistical principles. This distribution is then modified on the basis of deterministic geological data $\mathcal{O}_{\text {geo }}$ from well logs, core samples, and seismic signals, for example. The observations modify the prior distribution and define a posterior distribution $p_{1}\left(m \mid \mathcal{O}_{\text {geo }}\right)$ for the geostatistics model. At this point, we consider the flow information. The posterior distribution constructed on the basis of direct geological observations and principles becomes a new prior distribution $p_{2}(m)=p_{1}\left(m \mid \mathcal{O}_{\text {geo }}\right)$ for inference relative to observations $\mathcal{O}_{\text {fluid }}$ based on the observed flow history. The fluid observations further limit the geostatistical ensemble, and thus give rise to a second posterior distribution, $p_{2}\left(m \mid \mathcal{O}_{\text {fluid }}\right)=p_{1}\left(m \mid \mathcal{O}_{\text {fluid }}, \mathcal{O}_{\text {geo }}\right)$.

To define the probability framework more explicitly we suppose that the geology is given as a random field, on the basis of geostatistical models [9, 43, 44, 45]. These models define our prior distribution $p(m)$. For example, the permeability tensor $K$ may be given as a $\log$ normal random field, so that $\phi(\vec{x})=\ln K(\vec{x})$ is a Gaussian random field, uniquely determined by its mean and covariance, or variogram. The total flow for two-phase displacement is governed by Darcy's law

$$
\vec{v}(\vec{x})=-K \lambda \nabla p, \quad \nabla v=0
$$

where $\lambda$ is the total relative mobility. Since the coefficient $K$ in the flow equation is random, the resulting porous media flow is stochastic. Two-phase flow has an additional constitutive function that also depends on the geology, the fractional flow $f$. This is also a random field, and introduces additional randomness into the flow solutions. Thus, $f$ is the flux for the relative saturation $s$ of one of the phases in the Buckley-Leverett 
equation, whose simplest expression has the form

$$
\frac{\partial s}{\partial t}+\vec{v} \cdot \nabla f(s, \vec{x})=0 .
$$

An efficient solution algorithm $s_{a}(m)$ is based on a solution to the scale-up problem. The scale-up problem is to find an equivalent coarse grid, or meso-physics description of the flow through local spatial averaging of the fine grid (micro-physics) geological heterogeneities and flow equations.

In a series of papers $[19,35,27,28,5]$ devoted to the computation of effective dispersivity for transport of a passive scalar $(f(s)=s$ in $(29))$, the anomalous, or travel distance dependence, of the effective dispersivity was traced to a multiscale structure in the heterogeneity fields defining $K$. A number of quantitative relations were derived relating travel distance dependence of the dispersivity to the multiple scales in $K$. For nonlinear $f$, the same analysis has been applied $[5,26,20]$, with the conclusion that the heterogeneity-induced dispersion traveled with a speed approximately linear in $t$. This being the case, the effects of heterogeneity can be modeled better by modification of $f$ than by changes in effective dispersivity [26].

Consistent with this discussion, and following the ideas of $\S 2$, we introduce averaged equations [26]. To model single-phase flow, we set $\lambda=\mu^{-1}$, the inverse fluid viscosity, in (28). Then the averaged version of $(28)$ is

$$
\bar{v}=-\overline{\frac{K}{\mu} \nabla p}, \quad \nabla \cdot \bar{v}=0 .
$$

This equation is not useful as it stands, due to the fact that the right-hand side of the first equation in (30) is not a defined quantity. With $K$ and $\nabla p$ correlated statistically, as is normally the case, $\overline{K \nabla p} \neq \bar{K} \nabla \bar{p}$. To accommodate this fact, we introduce the quantity

$$
K_{\mathrm{ren}}=\frac{\overline{K \nabla p}}{\nabla \bar{p}}
$$

so that

$$
K_{\text {ren }} \nabla \bar{p}=\overline{K \nabla p}
$$

is a mathematical identity, and the averaged single-phase Darcy's law can be written as

$$
\bar{v}=-\frac{K_{\mathrm{ren}}}{\mu} \nabla \bar{p}
$$

Here we have used the fact that $\nabla$ is a linear operator; all linear operators commute with averages, so that $\overline{\nabla p}=\nabla \bar{p}$.

Equation (32) only rearranges our ignorance. The essential step is closure, which is to find an approximate expression for $K_{\text {ren }}$ in terms of the basic averaged dependent variables of the problem, and which can be substituted for definition (31). Specifically, $K_{\text {ren }}$ in (31) depends on the flow boundary conditions, whereas we require a definition, or approximate evaluation, independent of boundary conditions.

A standard solution to this problem is to define $K_{\text {ren }}$ using solutions corresponding to a specific choice of boundary conditions, e.g., periodic in $\nabla p$, with $p$ decreasing across this periodic domain in one of the coordinate directions. Three such problems, one for 
each of the three coordinate directions, uniquely determine $K_{\text {ren }}$ as a tensor. It has been demonstrated that this definition of $K_{\text {ren }}$ applies with satisfactory accuracy for other flow boundary conditions [15].

We retain this definition of $K_{\text {ren }}$ for two-phase flow and apply the same method to the definition of $f$ and $\lambda$. Thus

$$
\lambda_{\text {ren }}=\frac{\overline{K \lambda \nabla p}}{K_{\text {ren }} \nabla \bar{p}}
$$

and

$$
f_{\text {ren }}=\overline{v f} / \bar{v} .
$$

With these definitions, the averaged two-phase flow equations become

$$
\bar{v}=-K_{\text {ren }} \lambda_{\text {ren }} \nabla \bar{p} ; \quad \nabla \cdot \bar{v}=0
$$

and

$$
\bar{s}_{t}+\bar{v} \nabla f_{\text {ren }}=0 \text {. }
$$

As above, the critical step is not the mathematical identities in (35)-(36) but the closure step, whereby $f_{\text {ren }}$ and $\lambda_{\text {ren }}$ are approximated by functions of the appropriate averaged variables, in this case $\bar{s}$. In the case of local spatial averages, as opposed to ensemble averages in the case of stationary statistics, the renormalized functions can still depend on the locally averaged spatial variables, $\bar{x}, \bar{y}$, and $\bar{z}$. Thus we seek functions $K_{\text {ren }}(\bar{x}, \bar{y}, \bar{z}), \lambda_{\text {ren }}(\bar{s}, \bar{x}, \bar{y}, \bar{z})$, and $f_{\text {ren }}(\bar{s}, \bar{x}, \bar{y}, \bar{z})$ that satisfy (31) and (33)-(34) approximately. The proper choice of up-scaled $K_{\text {ren }}, \lambda_{\text {ren }}$, and $f_{\text {ren }}$ can be viewed as a closure problem, whose solution is dependent on the parameters that fix the geological ensemble of microphysical $K, k$, and $f[11]$.

We have used a mixture of mathematical theory, modeling, and DNS simulation to study the effect of averaging over the fine details in the problem specification.

Our scale-up method offers potential improvements [62] in simulation speed of perhaps $10^{4}$ in three space dimensions. This estimate is based on a rather extensive series of exploratory calculations. In these calculations, geological data was specified on a uniform fine grid. The fluid flow was computed on both the fine grid and on a grid that was coarsened by a factor of 10 in each dimension. Successful scale-up must be accurate as well as computationally efficient; so the coarse grid flow simulations must give approximately the same predictions as the finely gridded ones for important flow variables, such as flow breakthrough times and oil production curves. See Fig. 5, in which a finely gridded simulation (conceptually regarded as "exact") is compared with three scale-up strategies. The successful strategy is based on a non-uniformly coarsened grid with scale-up of both the absolute and relative permeabilities.

5.2. The inverse problem of parameter determination. Further modification of the ensemble of geologies is based on observations $\mathcal{O}$ of the fluid flow, including oil production rates and well pressure records. History matching is the search for geology realizations in agreement with available flow observations.

The methods proposed for solution of the inverse problem fall mainly into two families: deterministic methods such as gradient descent, which find a local maximum for the 


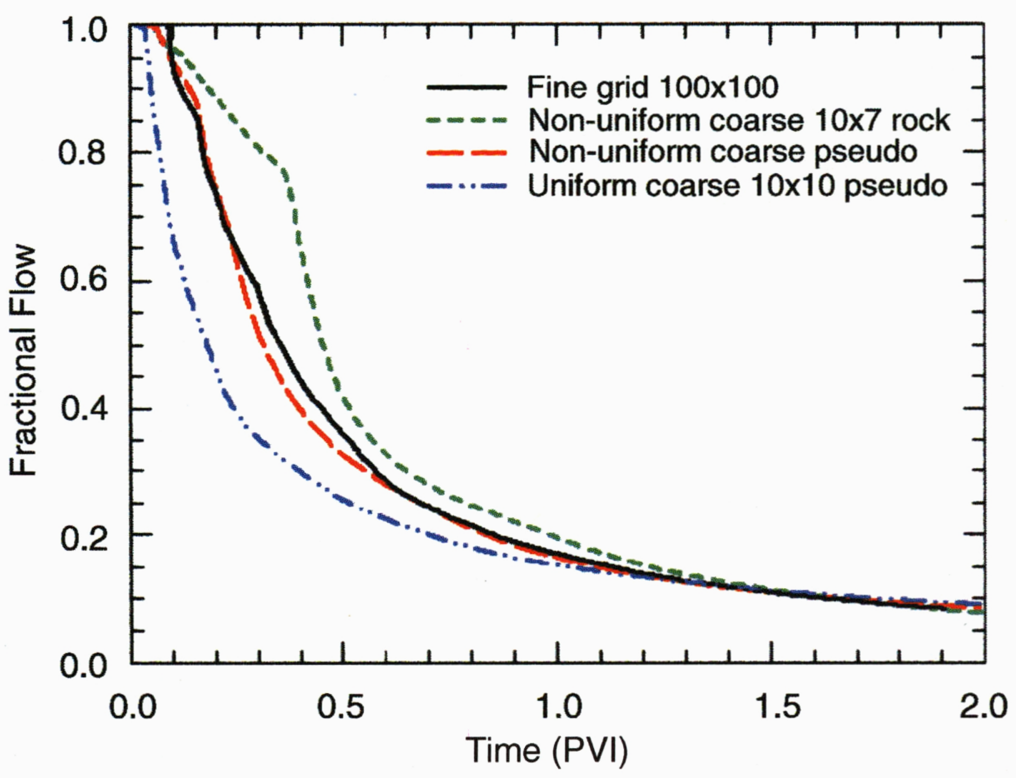

FIG. 5. Scale-up of flow in porous media by an average factor of 10 in each linear dimension. Fractional production of oil (relative to total fluid outflow) is plotted vs. time. The fine grid solution (solid curve) is regarded as exact. It is compared to three scale-up strategies, the preferred of which (dashed red curve) is based on a non-uniformly coarsened grid and scale-up of the absolute permeability $K$ as well as the relative permeability $k$ and the fractional flow $f[62]$.

posterior probability density with possible help from solution of the adjoint equation, and stochastic methods, such as simulated annealing and MCMC, the former of which finds a global maximum likelihood while the latter samples from and integrates over the posterior distribution.

The MCMC framework yields a comprehensive and predictive probability model for reservoir performance. Starting from a given geostatistical model (the prior distribution), the probability model will be conditioned on additional information. Information relating to the fluid flow comes from pressure and saturation production data accumulated over time at each well, and special tests performed at individual wells, such as well pressure tests.

Given a rapid approximate solution to the forward simulation problem, stochastic simulation to solve the inverse problem is feasible. Typical well-based flow history data allows inference of zone-averaged permeability values over a length scale close to the inter-well spacing [61]. Stochastic simulation methods typically have a higher error in predicting point values for log permeability than traditional maximum likelihood methods such a Kriging, [16], but are nonetheless more satisfactory, since they better represent statistically the high permeability channels that have a dominant influence on flow. Thus we can use a Kriging methodology, not to give a maximum likelihood realization of the geology, but rather to give a constrained mean and variance consistent with observed 
data, and sample from the resulting conditioned ensemble. One version of these ideas is called sequential Gaussian simulation [14]. Full Monte Carlo simulation yields the same result, when applied to a Gaussian random variable. While the log permeability is approximately normal for many porous media, the Monte Carlo method does not assume a Gaussian field, and is thus more general. See also [13]. In [58], genetic algorithms are compared to simulated annealing for solution of the inverse problem, while in [53], MCMC methods are studied. In [53], the more fundamental rigor of the statistical formulation of MCMC was explained, in contrast to simulated annealing, for example, which either yields a spurious unique solution to the inverse program, or depends in a phenomenological manner on the final stopping temperature to achieve an ensemble of solutions. This paper also makes the point that the linear (Gaussian) portion of the statistical problem can be solved exactly, by a change of variables to normal modes, in which the sampling of independent random Gaussian variables can be achieved by elementary means, and with Metropolis acceptance factors of one for new choices. This linear conditioning can be applied to well logging and similar geostatistical conditioning data. It also applies to a linearization of the nonlinear forward simulation, and to simulation output data, such as pressures and flow, which are nonlinearly related to the geostatistical data. This linearization goes under the name of sensitivity analysis, here used systematically in the selection of the next MCMC sample realization of the reservoir.

\section{REFERENCES}

[1] K. Abbaspour, R. Schulin, M. Th. van Genuchten, and E. Schlappi, Accounting for uncertainty in geostatistical parameters within a stochastic simulation, Technical report, Swiss Federal Institute of Technology, Department of Soil Protection, 1997

[2] K. Abbaspour, R. Schulin, M. Th. van Genuchten, and E. Schlappi, Procedures for uncertainty analysis applied to a landfill leacheate plume, Technical report, Swiss Federal Institute of Technology, Department of Soil Protection, 1997

[3] U. Alon, J. Hecht, D. Ofer, and D. Shvarts, Power laws and similarity of Rayleigh-Taylor and Richtmyer-Meshkov mixing fronts at all density ratios, Phys. Rev. Lett. 74, 534-538 (1995)

[4] U. Alon and D. Shvarts, Two-phase flow model for Rayleigh-Taylor and Richtmyer-Meshkov mixing, in R. Young, J. Glimm, and B. Boston, editors, Proceedings of the Fifth International Workshop on Compressible Turbulent Mixing, World Scientific, Singapore, 1996

[5] L. An, J. Glimm, D. H. Sharp, and Q. Zhang, Scale up of flow in porous media, in A. Burgeat, C. Carasso, S. Luckhaus, and A. Mikelic, editors, Mathematical Modeling of Flow Through Porous Media, World Scientific, 1995, pp. 26-44

[6] R. C. Bissel, Y. Sharma, and J. E. Killough, History matching using the method of gradients: Two case studies, SPE 28590, 1994. 69th Annual Technical Conference and Exhibition, New Orleans, 25-28 September, 1994

[7] Y. Chen, J. Glimm, D. H. Sharp, and Q. Zhang, A two-phase flow model of the Rayleigh-Taylor mixing zone, Phys. Fluids 8(3), 816-825 (1996)

[8] I.-L. Chern and T.-P. Liu, Convergence to diffusion waves of solutions for viscous conservation laws, Comm. Math. Phys. 110, 503-517 (1987)

[9] G. Christakos, Random Field Models in Earth Sciences, Academic Press, New York, 1992

[10] M. Christie, Private communication, 1997

[11] M. A. Christie and P. J. Clifford, A fast procedure for upscaling in compositional simulation, SPE 37986, 1997

[12] S. Cohn, An introduction to estimation theory, J. Meteorological Soc. Japan 77, 257-288 (1997)

[13] A. Datta-Gupta, L. W. Lake, and G. A. Pope, Characterizing heterogeneous permeable media with spatial statistics and tracer data using sequential simulated annealing, Mathematical Geology 27, 763-787 (1995) 
[14] C. V. Deusch and A. G. Journel, Geostatistical Software Library and User's Guide, Oxford University Press, Oxford, 1992

[15] L. J. Durlofsky, R. A. Behrens, R. C. Jones, and A. Bernath, Scale up of heterogeneous three dimensional reservoir descriptions, SPE Proceedings (SPE 30709), October 1995. Presented at SPE Annual Technical Conference and Exhibition, Dallas, USA, 22-25 October 1995

[16] J. R. Eggleston, S. A. Rojstaczer, and J. J. Pierce, Identification of hydraulic conductivity structure in sand and gravel aquifers: Cape cod data set, Water Res. Research 32, 1209-1222 (1996)

[17] J. T. Fredrich and W. B. Lindquist, Statistical characterization of the three-dimensional microgeometry of porous media and correlation with macroscopic transport properties, Internat. J. Rock Mech. and Min. Sci. 34, 3-4 (1997)

[18] N. Freed, D. Ofer, D. Shvarts, and S. Orszag, Two-phase flow analysis of self-similar turbulent mixing by Rayleigh-Taylor instability, Phys. Fluids A 3(5), 912-918 (1991)

[19] F. Furtado, J. Glimm, W. B. Lindquist, and F. Pereira, Multi length scale calculations of mixing length growth in tracer floods, in F. Kovarik, editor, Proceedings of the Emerging Technologies Conference, Institute for Improved Oil Recovery, Univ. Houston, Houston, TX, 1990, pp. 251-259

[20] F. Furtado and F. Pereira, Scaling analysis for two-phase immiscible flow in heterogeneous porous media, Technical report, University of Wyoming, 1997

[21] Alan E. Gelfand and Adrian F. M. Smith, Marginal densities, J. American Statistical Association 85, 398, 409 (1990)

[22] S. Geman and D. Geman, Stochastic relaxation, Gibbs distributions and the Bayesian restoration of images, IEEE Transactions on Pattern Analysis and Machine Intelligence 6, 721-741 (1984)

[23] W. R. Gilks, S. Richardson, and D. J. Spiegelhalter, editors, Markov Chain Monte Carlo in Practice, Chapman and Hall, London and New York, 1996

[24] J. Glimm, J. Grove, X.-L. Li, and D. C. Tan, Robust computational algorithms for dynamic interface tracking in three dimensions, Technical Report, in preparation, 1998

[25] J. Glimm, J. W. Grove, X.-L. Li, K.-M. Shyue, Q. Zhang, and Y. Zeng, Three dimensional front tracking, SIAM J. Sci. Comp. 19 (1998) (to appear)

[26] J. Glimm, H. Kim, D. Sharp, and T. Wallstrom, A stochastic analysis of the scale up problem for flow in porous media, Computational and Applied Mathematics 17 (1998)

[27] J. Glimm, W. B. Lindquist, F. Pereira, and R. Peierls, The multi-fractal hypothesis and anomalous diffusion, Mat. Aplic. Comput. 11, 189-207 (1992)

[28] J. Glimm, W. B. Lindquist, F. Pereira, and Q. Zhang, A theory of macrodispersion for the scale up problem, Transport in Porous Media 13, 97-122 (1993)

[29] J. Glimm, D. Saltz, and D. H. Sharp, Renormalization group solution of two-phase flow equations for Rayleigh-Taylor mixing, Phys. Lett A, 222, 171-176 (1996)

[30] J. Glimm, D. Saltz, and D. H. Sharp, A general closure relation for incompressible mixing layers induced by interface instabilities, Technical Repoit SUNYSB-AMS-97-11, SUNY at Stony Brook, 1997. Submitted to Proceedings of the Sixth International Workshop on the Physics of Compressible Turbulent Mixing

[31] J. Glimm, D. Saltz, and D. H. Sharp, The statistical evolution of chaotic fluid mixing, Technical Report SUNYSB-AMS-97-05, SUNY at Stony Brook, 1997. Submitted to Phys. Rev. Lett.

[32] J. Glimm, D. Saltz, and D. H. Sharp, Two-phase modeling of a fluid mixing layer, Technical Report SUNYSB-AMS-97-01, SUNY at Stony Brook, 1997. Submitted to J. Fluid Mech.

[33] J. Glimm, D. Saltz, and D. H. Sharp, Two-pressure two-phase flow, in G.-Q. Chen, Y. Li, and X. Zhu, editors, Nonlinear Partial Differential Equations, World Scientific, 1998

[34] J. Glimm and D. H. Sharp, Chaotic mixing as a renormalization group fixed point, Phys. Rev. Lett. 64, 2137-2139 (1990)

[35] J. Glimm and D. H. Sharp, A random field model for anomalous diffusion in heterogeneous porous media, J. Stat. Phys. 62, 415-424 (1991)

[36] J. Glimm and D. H. Sharp, Stochastic partial differential equations: Selected applications in continuum physics, in R. A. Carmona and B. L. Rozovskii, editors, Stochastic Partial Differential Equations: Six Perspectives, Mathematical Surveys and Monographs, American Mathematical Society, Providence, RI, 1997

[37] J. Glimm and X.-L. Li, On the validation of the Sharp-Wheeler bubble merger model from experimental and computational data, Phys. Fluids 31, 2077-2085 (1988)

[38] J. Grove, R. Holmes, D. H. Sharp, Y. Yang, and Q. Zhang, Quantitative theory of RichtmyerMeshkov instability, Phys. Rev. Lett 71(21), 3473-3476 (1993) 
[39] W. K. Hastings, Monte Carlo sampling methods using Markov chains and their applications, Biometrika 87, 97-109 (1970)

[40] F. Hiller and G. Lieberman, Introduction to Operations Research, McGraw-Hill, New York, fifth edition, 1990

[41] J. H. Holland, Adaption in Natural and Artificial Systems, MIT Press, Cambridge, 1992

[42] R. L. Holmes, J. W. Grove, and D. H. Sharp, Numerical investigation of Richtmyer-Meshkov instability using front tracking, J. Fluid Mech. 301, 51-64 (1995)

[43] A. G. Journel and Ch. J. Huijbregts, Mining Geostatistics, Academic Press, New York, 1978

[44] L. Lake and H. Carroll, editors, Reservoir Characterization, Academic Press, New York, 1986

[45] L. Lake, H. Carroll, and T. Wesson, editors, Reservoir Characterization II, Academic Press, New York, 1991

[46] J. Lam and J.-M. Delosme, An efficient simulated annealing schedule: Derivation, Report No. 8816, Yale Electrical Engineering Department, New Haven, CT, 1988

[47] J. Lam and J.-M. Delosme, An efficient simulated annealing schedule: Implementation and evaluation, Report No. 8817, Yale Electrical Engineering Department, New Haven, CT, 1988

[48] C. Leith, Nonlinear normal-mode initialization of numerical weather prediction models, in J. Brackbill and B. Cohen, editors, Multiple Time Scales, Academic Press, New York, 1985, pp. 59-71

[49] W. B. Lindquist, S.-M. Lee, D. A. Coker, K. W. Jones, and P. Spanne, Medial axis analysis of void structure in three-dimensional tomographic images of porous media, J. Geophys. Res. 101B, 8297-8310 (1996)

[50] D. Mihalas and B. Mihalas, Foundations of Radiation Hydrodynamics, Oxford Univ. Press, New York, 1984

[51] R. Mulder, S. Osher, and J. A. Sethian, Computing interface motion in compressible gas dynamics, J. Comp. Phys. 100, 209-228 (1992)

[52] J. T. Oden and T. I. Zohdi, Analysis and adaptive modeling of highly heterogeneous elastic structures, Technical Report TICAM Report 96-56, University of Texas at Austin, 1996

[53] D. S. Oliver, L. B. Cunha, and A. C. Reynolds, Markov chain Monte Carlo methods for conditioning a permeability field to pressure data, Math. Geo. 29, 61-91 (1997)

[54] B. Plohr and D. Sharp, Instability of accelerated elastic metal plates, Report No. SUNYSB-AMS97-13, State Univ. of New York at Stony Brook, 1997

[55] S. Kirkpatrick, C. D. Gelatt, and M. P. Vecchi, Optimization by simulated annealing, Technical report, 1982

[56] M. B. Schneider, G. Dimonte, and B. Remington, Large and small scale structure in Rayleigh-Taylor mixing, Phys. Rev. Lett., 1997, Submitted

[57] G. A. F. Seber, Multivariate Observations, John Wiley and Sons, New York, 1984

[58] M. K. Sen, A. Datta-Gupta, P. L. Stoffa, L. Lake, and G. A. Pope, Stochastic reservoir modeling using simulated annealing and genetic algorithms, SPE Formation and Evaluation 10, 49-56 (1995)

[59] D. M. Snider and M. J. Andrews, Rayleigh-Taylor and shear driven mixing with an unstable thermal stratification, Phys. Fluids 6(10), 3324-3334 (1994)

[60] P. Spanne, J. F. Thovert, C. J. Jacquin, W. B. Lindquist, K. W. Jones, and P. M. Adler, Synchrotron computed microtomography of porous media: Topology and transports, Phys. Rev. Lett. 73, 20012004 (1994)

[61] D. W. Vasco and A. Datta-Gupta, Integrating multiphase production history in stochastic reservoir characterization, SPE Formation and Evaluation 12, 149-156 (1997)

[62] T. Wallstrom, S. Hou, D. H. Sharp, M. A. Christie, and L. J. Durlofsky, Improved reservoir simulation using upscaled relative permeabilities and flexible grids (in preparation)

[63] Y. Yang, Q. Zhang, and D. H. Sharp, Small amplitude theory of Richtmyer-Meshkov instability, Phys. Fluids 6(5), 1856-1873 (1994)

[64] D. L. Youngs, Modeling turbulent mixing by Rayleigh-Taylor instability, Physica D, 37, 270-287 (1989)

[65] D. L. Youngs, Three-dimensional numerical simulation of turbulent mixing by Rayleigh-Taylor instability, Phys. Fluids A, 3, 1312-1319 (1991)

[66] Q. Zhang, Validation of the chaotic mixing renormalization group fixed point, Phys. Lett. A, 151, $18-22(1990)$

[67] Q. Zhang and M. J. Graham, A numerical and theoretical study of Richtmyer-Meshkov instability driven by cylindrical shocks, Report No. SUNYSB-AMS-96-15, State Univ. of New York at Stony Brook, 1996 
[68] Q. Zhang and M. J. Graham, Scaling laws for unstable interfaces driven by strong shocks in cylindrical geometry, Physical Review Letters, 1997, Submitted

[69] Q. Zhang and S. Sohn, An analytical nonlinear theory of Richtmyer-Meshkov instability, Phys. Lett. A, 212, 149-155 (1996)

[70] Q. Zhang and S. Sohn, Padé approximation to an interfacial fluid mixing problem, Applied Mathematics Letters 10, 121-127 (1997)

[71] Q. Zhang and S. Sohn, Nonlinear solutions of unstable fluid mixing driven by shock waves, Physics of Fluids, 1997, Accepted

[72] T. I. Zohdi, J. T. Oden, and G. J. Rodin, Hierarchical modeling of heterogeneous bodies, Comput. Methods Appl. Mech. Engrg. 138, 273-298 (1996) 